\title{
Erratum: Notch signaling controls the generation and differentiation of early T lineage progenitors
}

Arivazhagan Sambandam, Ivan Maillard, Valerie P Zediak, Lanwei Xu, Rachel M Gerstein, Jon C Aster, Warren S Pear \& Avinash Bhandoola Nature Immunology 6, 663-670 (2005)

On page 664, a panel in Figure 3a was missing the value '0.2' beside the outlined area. The figure with correct labeling is provided here.
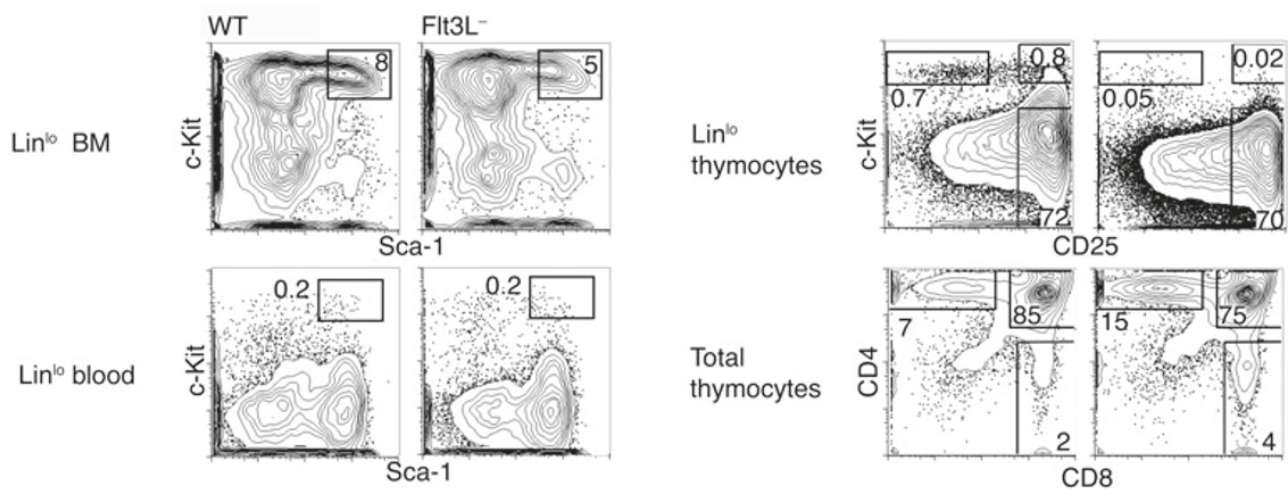

\section{Corrigendum: A critical function for type I interferons in cancer immunoediting}

Gavin P Dunn, Allen T Bruce, Kathleen C F Sheehan, Vijay Shankaran, Ravindra Uppaluri, Jack D Bui, Mark S Diamond, Catherine M Koebel, Cora Arthur, J Michael White \& Robert D Schreiber Nature Immunology 6, 722-729 (2005); corrected 24 June 2005

On page 724, the lines in the graphs in Figure 4a,c should be labeled 'WT'. The figure with correct labeling is provided here.
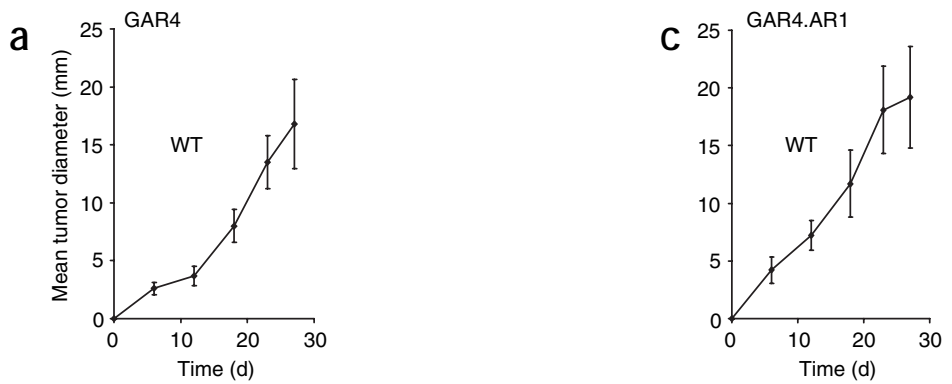